\title{
Efficient route for the synthesis of diverse heteroannelated 3-cyanopyridines
}

\author{
A. Mityuk, S. Ryabukhin, D. Volochnyuk
}

\section{Introduction and Aim}

Fused pyridines become more and more interesting for researchers involved in drug-design and medicinal chemistry. This class of compounds has already found use as antidepressants, antiallergics, antidiabetics, antihistaminics, etc. A significant part of hits are heterocycles connected to pyridine core. Today a lot of different methods of synthesis are developed, but many questions to discuss still have been left. For example, the efficient synthesis of hetero annelated 5-cyanopyridines in one step using sodium 2-cyano-3, 3-dimethoxyprop-1-en-1-olate was described 30 years ago. Yet, the scope of the reaction for different heterocycles and the possibility of scale up to multi grams had not been shown. Subsequent studies didn't look to this reaction more profoundly. In our research, we focus the attention on the expansion of scope and limitation of this reaction to diverse heterocycles and adaptation of the synthetic procedure to scale up.

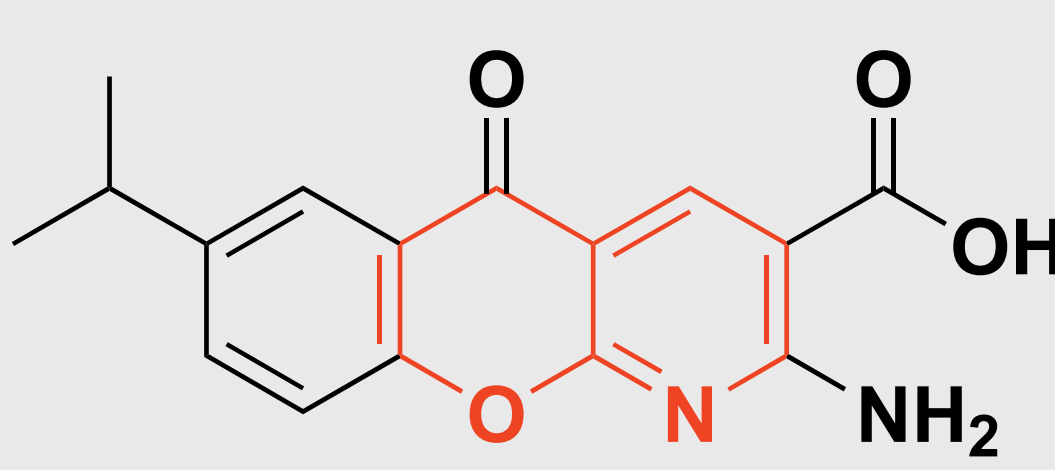

Amlexanox

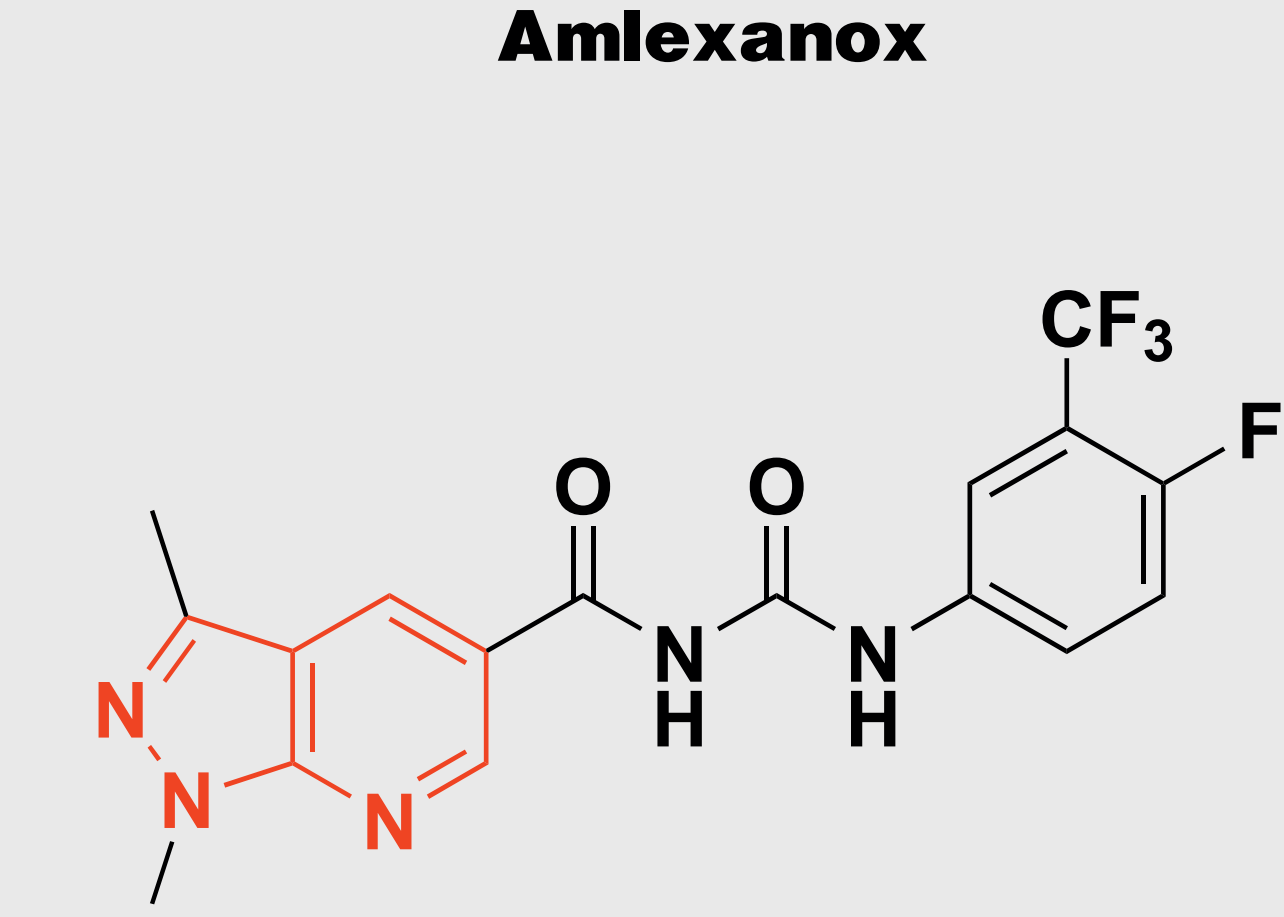

TLK-19705

autoimune diaseses
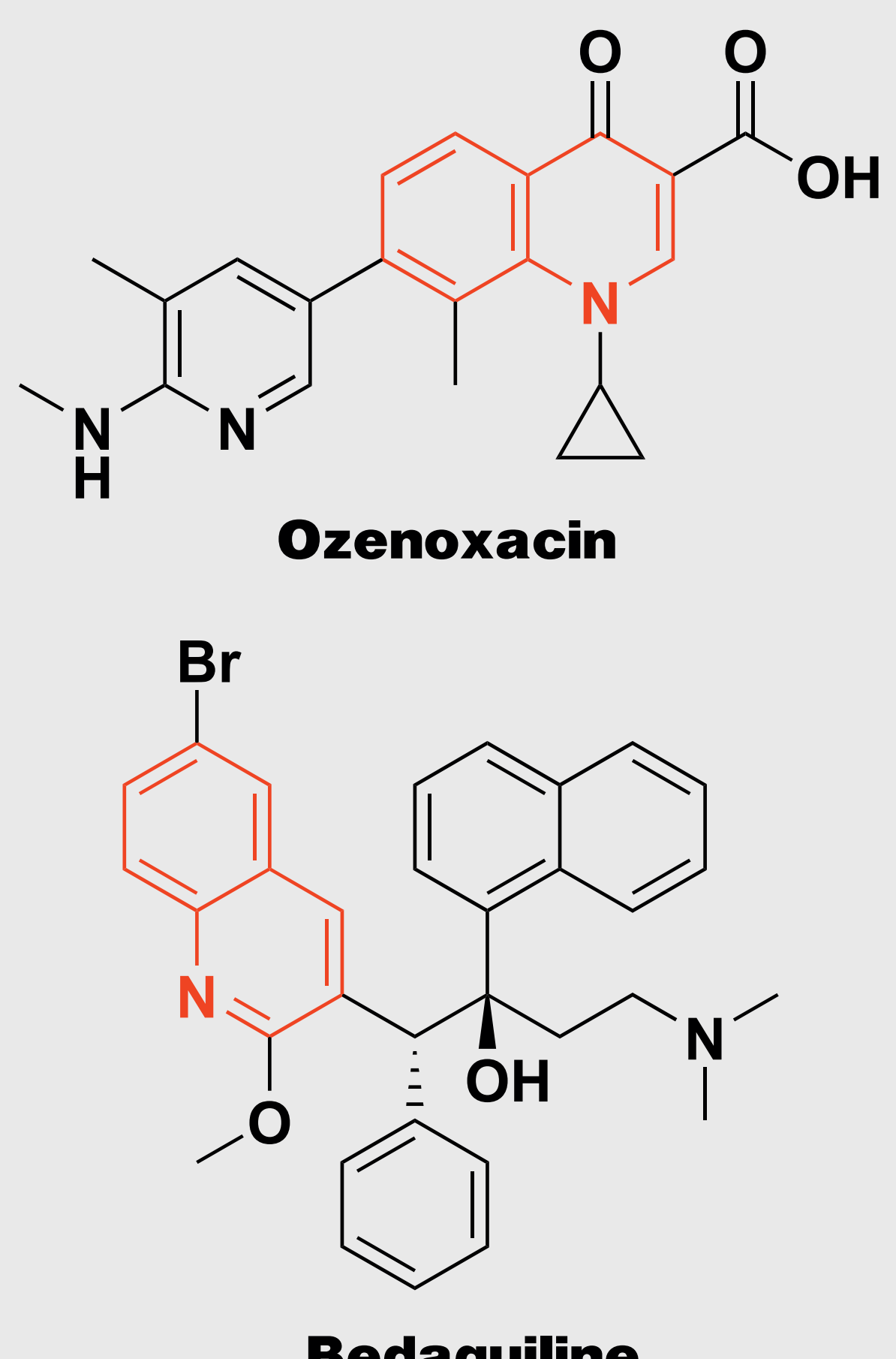

the procedure are determined and will be discussed. A The synthesis of starting reagent sodium 2-cyano-2- number of diverse hetero annelated 5-cyanopyridines, the (dimethoxymethyl) eth-1-en-1-olate in kilo scale is elaborated. The promising building blocks for Medicinal chemistry, were synthetic methodology for the cyclization is validated and optimized for synthesized in multi grams. more than $50 \mathrm{~g}$ scales. The advantages and scope and limitations of

\section{Synthesis}

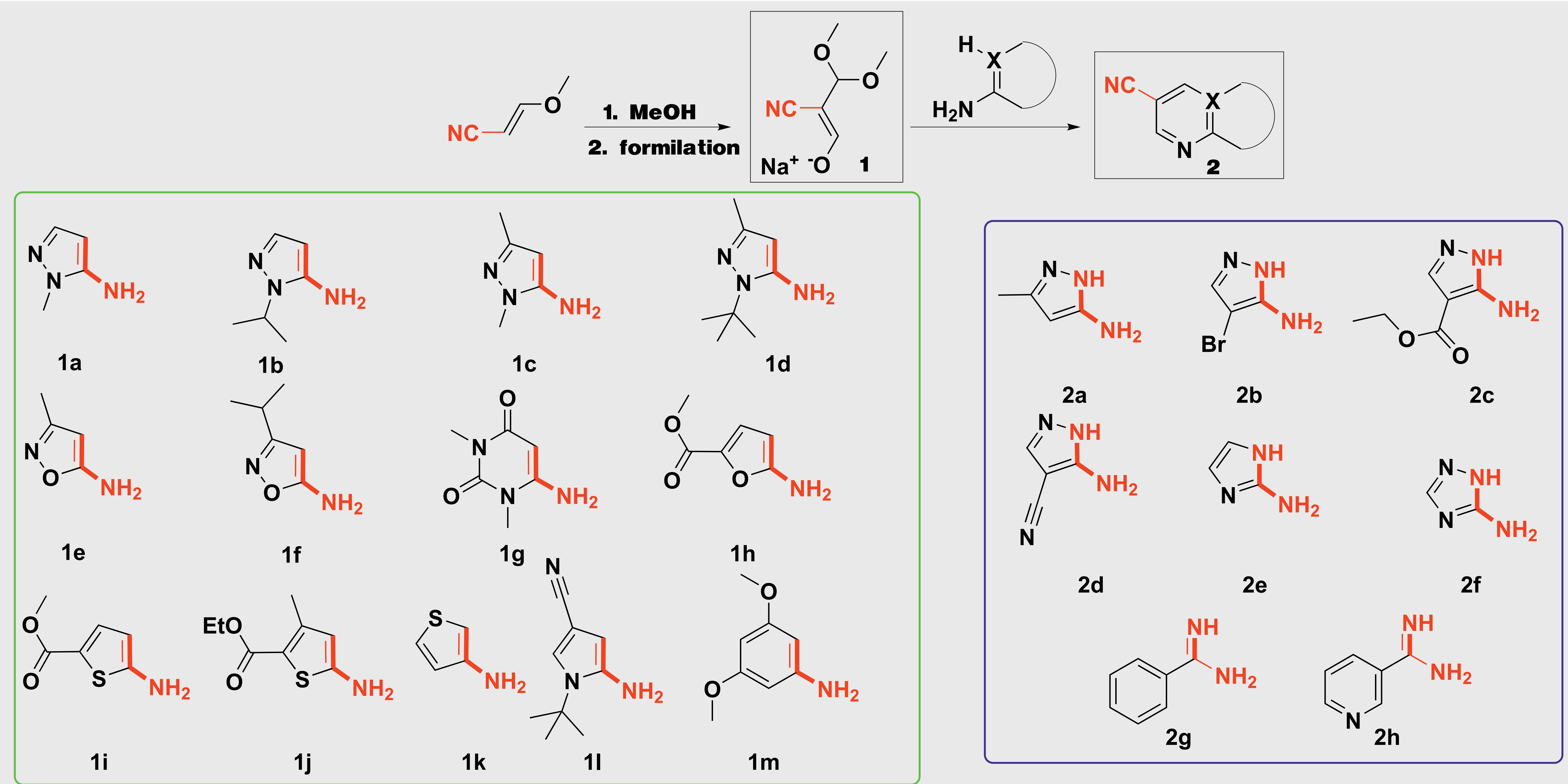

\section{Results}

It was shown that sodium 2-cyano-2-(dimethoxymethyl)eth-1-en-1-olate can be used as a reagent to obtain easily 5-cyanopyridine fused with various heterocycles. This method works with $\mathrm{CCN}$-nucleophiles as well as with NCN-nucleophiles, and if the substrate can react both ways reaction will give NCN-product.

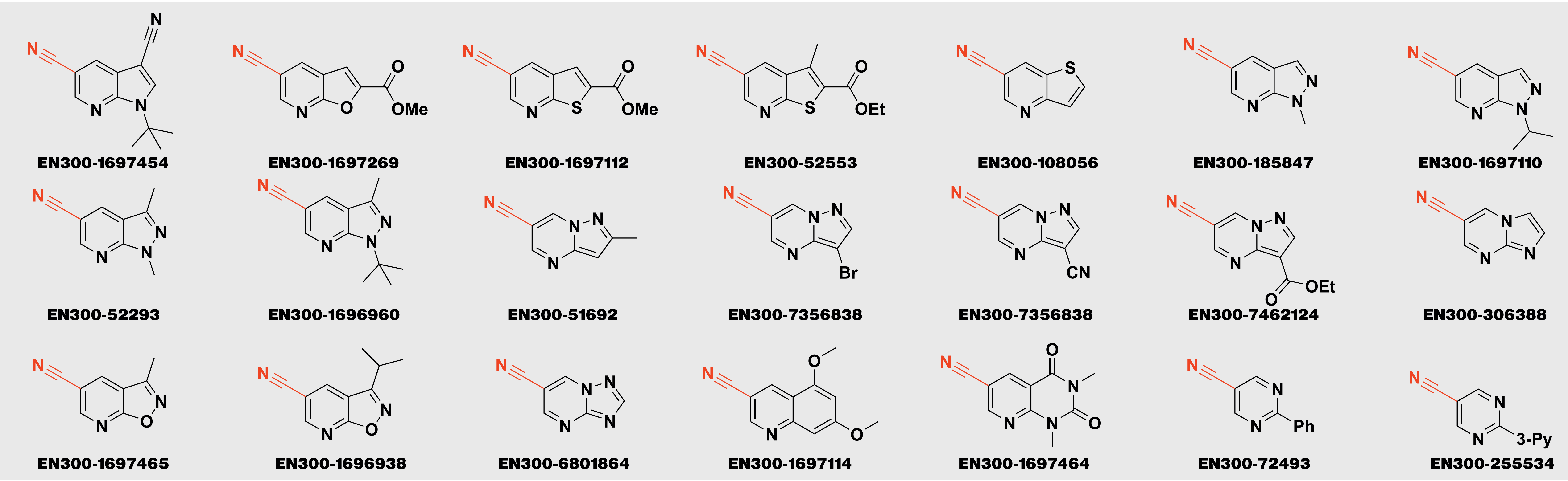

